\title{
Magnetization and Giant Magnetoimpedance Effect of Co-Rich Microwires under Different Driven Currents
}

\author{
Shengdi Sun, ${ }^{1}$ Shuling Zhang, ${ }^{2,3}$ Bo Zhang, ${ }^{2}$ and Wenjie Ding ${ }^{2}$ \\ ${ }^{1}$ School of Mechanical and Electrical Engineering, Heilongjiang Institute of Technology, Heilongjiang 150001, China \\ ${ }^{2}$ School of Mechanical Engineering, Ningxia University, Ningxia 750021, China \\ ${ }^{3}$ School of Materials Science and Engineering, Taiyuan University of Science and Technology, Shanxi 030024, China \\ Correspondence should be addressed to Shuling Zhang; slzhang1229@163.com
}

Received 25 June 2015; Revised 12 September 2015; Accepted 27 September 2015

Academic Editor: Liling Fu

Copyright (C) 2016 Shengdi Sun et al. This is an open access article distributed under the Creative Commons Attribution License, which permits unrestricted use, distribution, and reproduction in any medium, provided the original work is properly cited.

$\mathrm{Co}_{68.25} \mathrm{Fe}_{4.5} \mathrm{Si}_{12.25} \mathrm{~B}_{15}$ amorphous microwires with a diameter of $34 \mu \mathrm{m}$ were prepared via the melt extraction method. The dependency of AC driving current $I_{\mathrm{ac}}$ and frequency on giant magnetoimpedance (GMI) effect and magnetization were investigated using a 4294A impedance analyzer and the transverse Kerr effect. The GMI effect was analyzed when $I_{\text {ac }}$ changed from $6 \mathrm{~mA}$ to $20 \mathrm{~mA}$ at a frequency ranging from $0.1 \mathrm{MHz}$ to $15 \mathrm{MHz}$. The influence of AC current dependent on the frequency is correlated with the magnetization mechanism. The maximum transverse Kerr intensity (MTKI) decreased with the increase in $I_{\text {ac }}$ under direct magnetic field when the frequency was below megahertz. However, MTKI values were similar with the increase of $I_{\text {ac }}$ when it was over $2 \mathrm{MHz}$. Meanwhile, the GMI effect was optimized by selecting an adequate value of AC driving current $I_{p}$, at which the circular permeability was higher when the frequency was not over $2 \mathrm{MHz}$. Results showed that the influence of $I_{\mathrm{ac}}$ on magnetoimpedance became weak with strong skin effect and slightly stronger GMI effect driven by a higher $I_{\mathrm{ac}}$ when the frequency was between $2 \mathrm{MHz}$ and $15 \mathrm{MHz}$. The skin effect turned out to be the key factor to the GMI effect; thus, there were no obvious differences in magnetization and GMI effect with AC driving current changing when the frequency was as high as $15 \mathrm{MHz}$.

\section{Introduction}

Giant magnetoimpedance (GMI) effect refers to the strong and sensitive change of impedance of a magnetic conductor with a small variation in DC magnetic field $[1,2]$. This research has been fuelled due to its potential applications in magnetic field sensing technology, particularly for magnetic sensors [3-5]. The origin of GMI effect in microwires has been interpreted in terms of skin effect and variation in circumferential permeability caused by axially applied DC magnetic field. The frequency, $f$, of the AC current must be high enough considering the skin depth, which is typically above $100 \mathrm{kHz}$. The impedance, $Z$, for a magnetic wire is given by $[1,6]$

$$
Z=\frac{R_{\mathrm{DC}}(k r) J_{0}(k r)}{2 J_{1}(k r)}
$$

where $R_{\mathrm{DC}}$ is the direct resistance of microwire and $J_{0}$ and $J_{1}$ are the Bessel functions. $k$ is defined as $k=(1+j) / \delta, j$ is the imaginary unit, and $\delta$ is the penetration depth which is given by the following function:

$$
\delta=\frac{1}{\sqrt{\pi \sigma \mu_{\Phi} f}}
$$

where $\sigma$ is the electrical conductivity and $\mu_{\Phi}$ is the circular permeability. In GMI response, change of penetration depth is induced by the applied magnetic field through the modification of $\mu_{\Phi}$, thereby resulting in a change of impedance. Meanwhile, AC current results in a circumferential magnetic field, which influences $\mu_{\Phi}$ through modifying the magnetization process. In other words, GMI effect depends on the amplitude of the AC driving current and frequency [7]. In addition, the conspiration effect of frequency and AC current on the magnetization process was analyzed to 


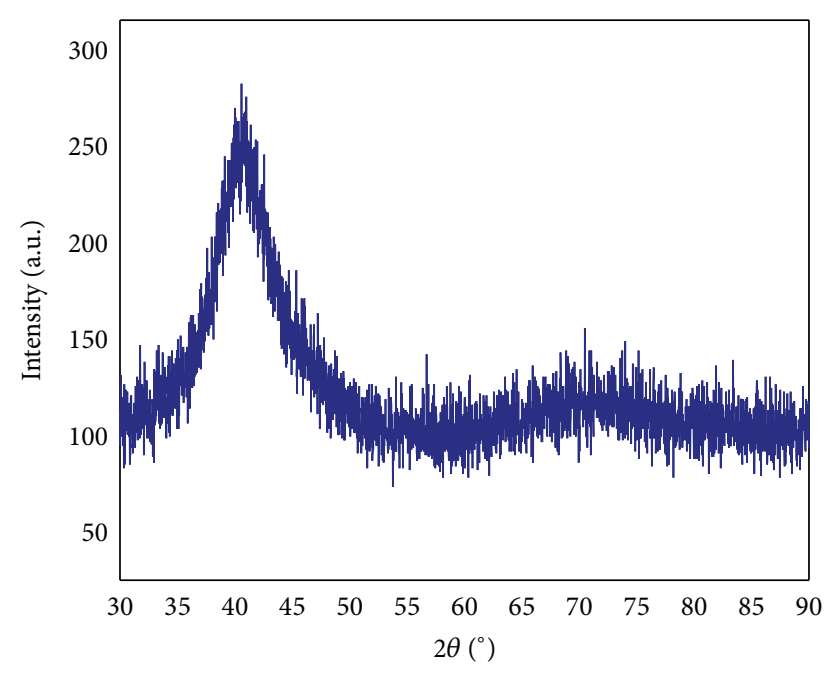

FIGURE 1: The XRD patterns of Co-rich microwires.

study the influence of AC current on the GMI effect. The majority of GMI studies to date are focused on Co-rich microwires fabricated via the Taylor wire technique [810]. Furthermore, melt extraction method has recently been revisited with a new technique, which yields high-quality microwires with superior soft magnetic properties compared with other approaches, such as in-rotating water spinning, Taylor wire, and glass-coated melt spinning. The excellent soft magnetic property and uniform diameter are the advantages of melt-extracted wires, making them more suitable for sensor applications without glass cover. In addition, their GMI effect is also excellent [11]. The stability of GMI effect under different AC currents $I_{\mathrm{ac}}$ was better and the fluctuations almost disappeared when $I_{\mathrm{ac}}$ was over $5 \mathrm{~mA}$ [12].

In this study, the dependency of AC driving current on magnetization and GMI effect is investigated in a frequency range of $0.1 \mathrm{MHz}$ to $15 \mathrm{MHz}$. A rich variety of multipeak features in GMI response depended on the driving current within a small DC magnetic field range, which have been observed and discussed.

\section{Experimental Details}

$\mathrm{Co}_{68} \mathrm{Fe}_{4.5} \mathrm{Si}_{15} \mathrm{~B}_{12.5}$ amorphous wires were prepared using the melt extraction method. The X-ray diffraction was done with microwires paralleled on glass holders. And it is found that microwires with a diameter below $50 \mu \mathrm{m}$ are amorphous, as shown in Figure 1.

And microwires with a diameter of $34 \mu \mathrm{m}$ were selected for investigation and connected in holders. The GMI effect was measured at room temperature using an Agilent 4294A impedance analyzer at an AC current frequency ranging from $0.1 \mathrm{MHz}$ to $15 \mathrm{MHz}$, with its amplitude varying between 6 and $20 \mathrm{~mA}$. The magnetoimpedance ratio, $\Delta Z / Z$, can be expressed as

$$
\frac{\Delta Z}{Z}=\frac{\left[Z\left(H_{\mathrm{ex}}\right)-Z\left(H_{0}\right)\right]}{Z\left(H_{0}\right)} \times 100 \%,
$$

where $Z\left(H_{\mathrm{ex}}\right)$ and $Z\left(H_{0}\right)$ are impedances at external field of $H_{\text {ex }}$ and zero, $H_{0}$. And the maximum magnetic field $H_{\text {ex }}$ was $320 \mathrm{~A} / \mathrm{m}$ and supplied by a solenoid which was placed and fixed in a special iron box to shield the earth magnetic field. And there is a platform with $60 \mathrm{~mm} \times 60 \mathrm{~mm}$ in size in the center of the solenoid at which the magnetic field is stable. The holders with microwires were then blocked in this platform by four folders.

The Kerr effect measurements and setup [11] were made in Basque Country University. The polarized light of a $\mathrm{He}-\mathrm{Ne}$ laser was reflected from the wire to the detector. The intensity of the reflected light was proportional to the magnetization when the transverse Kerr effect was used, which was perpendicular to the plane on flight. An electric current flowing along the wire produced a circular magnetic field. An axial magnetic field was produced by a pair of Helmholtz coils.

\section{Results and Discussion}

Figure 2 shows the frequency dependency of magnetoimpedance $Z$ at different magnetic field, with $I_{\mathrm{ac}}$ varying from $6 \mathrm{~mA}$ to $20 \mathrm{~mA}$. The value of impedance increases with the increase of DC magnetic field $H_{\mathrm{ex}}$ and reaches its peak value, $Z_{m}$, when the external field is equal to the effective anisotropy field, $H_{m}$. An increase of impedance at zero magnetic field, $Z\left(H_{0}\right)$, accompanied by a decrease of $H_{m}$ is observed when the driving current $I_{\mathrm{ac}}$ is increased from $6 \mathrm{~mA}$ to $20 \mathrm{~mA}$. For example, at $0.2 \mathrm{MHz}, Z\left(H_{0}\right)$ increases from $40.9 \Omega$ to $55.6 \Omega$, and $H_{m}$ decreases from $51.2 \mathrm{~A} / \mathrm{m}$ to $40 \mathrm{~A} / \mathrm{m}$. However, the maximum $Z_{m}$ first increases from $55.7 \Omega$ to $64 \Omega$ at $9 \mathrm{~mA}$ and then decreases to $56.6 \Omega$ when $I_{\mathrm{ac}}$ increases to $20 \mathrm{~mA}$. The largest impedance ratio $\Delta Z / Z$ is up to $50.0 \%$ at this frequency, with an AC current $I_{\mathrm{ac}}$ of $9 \mathrm{~mA}$. Meanwhile, $Z\left(H_{0}\right)$ increases from $41.1 \Omega$ to $64.3 \Omega$ at $0.4 \mathrm{MHz}$, and the largest $Z_{m}$ of $75.6 \Omega$ is observed when $I_{\mathrm{ac}}$ was $12 \mathrm{~mA}$. When the frequency is over $0.6 \mathrm{MHz}, Z_{m}$ increases with the increase of AC current $I_{\mathrm{ac}}$. At the same frequency, the impedance $Z\left(H_{0}\right)$ is proportional to the square root of the circular permeability given by (1) and (2). Therefore, the variation in impedance value reflects the evolution of circular permeability with different AC currents.

Another different feature is also observed. GMI effect is better under driving current of $9 \mathrm{~mA}$, when the frequency ranges from $0.2 \mathrm{MHz}$ to $0.8 \mathrm{MHz}$, but GMI effect is better at $12 \mathrm{~mA}$ when the frequency is from $0.8 \mathrm{MHz}$ to $2 \mathrm{MHz}$. These results indicate the existence of optimal AC driving current $I_{p}$ at which GMI response is best at a certain frequency.

When frequency increases to several megahertz, the difference of impedance $Z\left(H_{0}\right), Z_{m}$, and $H_{m}$ with the AC amplitude gets comparatively stable with increase of frequency. When the frequency is as high as $15 \mathrm{MHz}$, the influence of driving current on impedance curves gets trivial. The impedance $Z\left(H_{0}\right)$ increases from $146.8 \Omega$ to $149.6 \Omega$, and $\Delta Z / Z$ increases from $213.9 \%$ to $214.9 \%$, where the difference of $H_{m}$ is negligible.

Furthermore, the GMI profile of these wires shows that there are two impedance peaks at 0.8 and $1 \mathrm{MHz}$ and three 


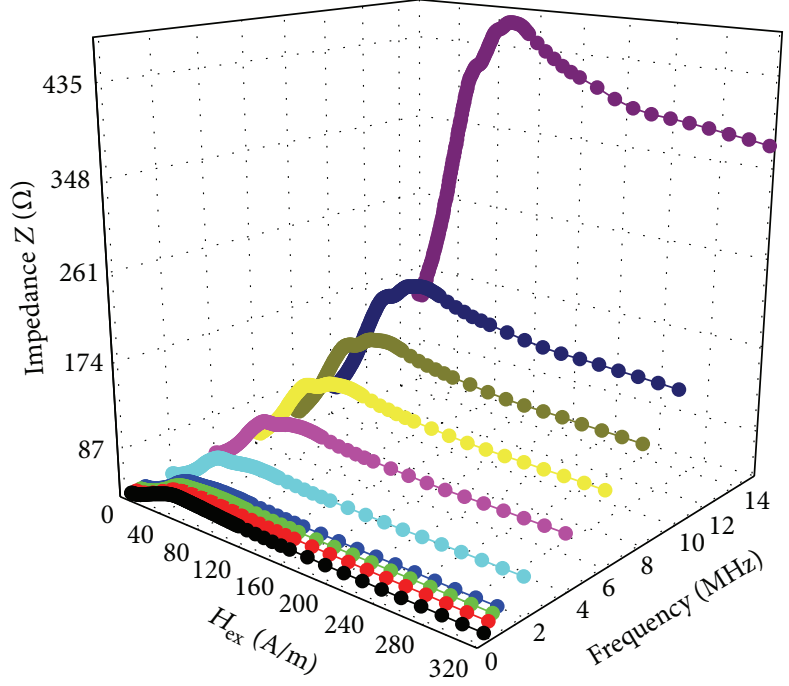

(a)

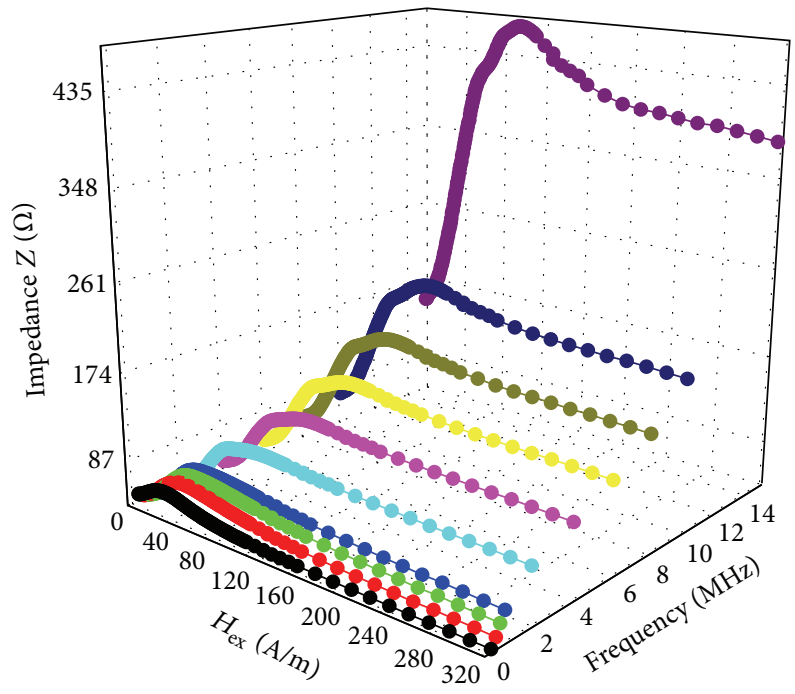

(c)

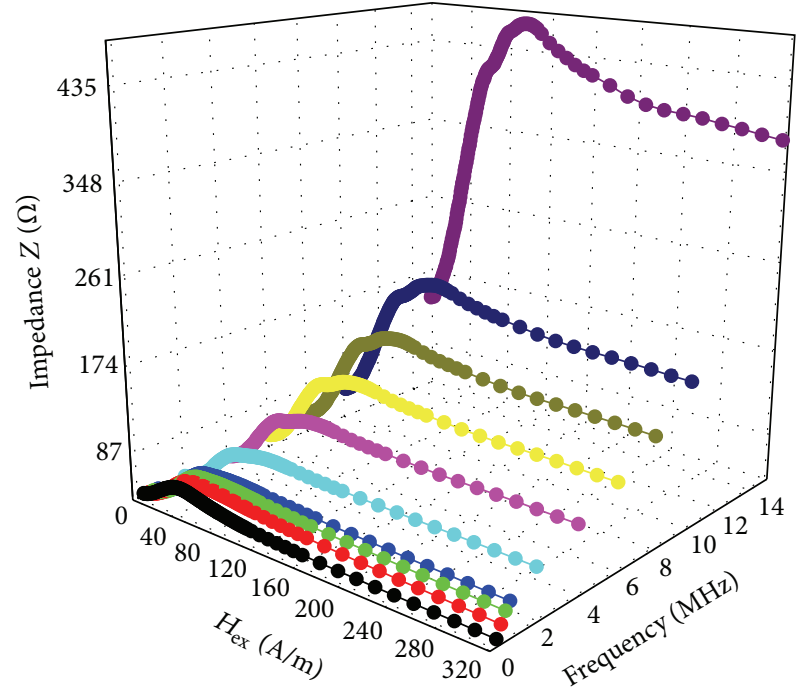

(b)

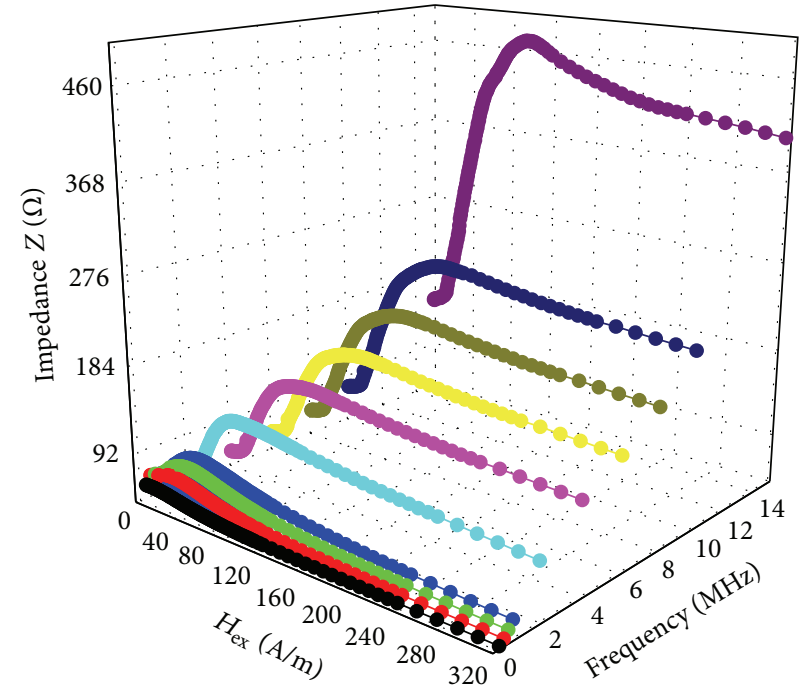

(d)

FIGURE 2: Field dependency of impedance at different frequencies and driving current: (a) $6 \mathrm{~mA}$, (b) $9 \mathrm{~mA}$, (c) $12 \mathrm{~mA}$, and (d) $20 \mathrm{~mA}$.

peaks over $2 \mathrm{MHz}$, driven by $6 \mathrm{~mA}$. At the same frequency, with driving current increasing, the second and third peaks move too close until gathering together and becoming one peak, which shows a clear driving current dependency. The multipeak feature is observed typical and much clearer when driving current is $2 \mathrm{~mA}$ in these Co-rich melt extraction microwires, as shown in Figure 3. As the AC frequency increases, the second and third peaks successfully appear at the back of the first peak, which also expresses a frequency dependency of multipeak feature when the driving current is $2 \mathrm{~mA}$. The evolution of multipeak features is associated with the circular magnetization process [2], and its amplitude sensitively evolves with the axial DC magnetic field, driving current amplitude, and frequency.

The variation in hysteresis loops with $\mathrm{AC}$ current reflects the change of magnetization reversal mechanism analyzed in detail in [13], and its effect on the circular permeability was also included [8]. The Kerr intensity is the intensity of the circular permeability in the shell from the loops [14]. Figure 4 presents the transverse Kerr intensity evolution of this Corich amorphous wire at $50 \mathrm{~Hz}$ under different AC driving current amplitudes. Maximum transverse Kerr intensity decreased from 0.144 to 0.02 at $50 \mathrm{~Hz}$, as shown in Figure 4(a) when the driving current increased from $0.5 \mathrm{~mA}$ to $6 \mathrm{~mA}$, indicating that the reduction of circular magnetization in DC magnetic field as AC current is increasing, which also proves the variation in circular permeability. Furthermore, coercivity $H_{c}$ decreased from $50.5 \mathrm{~A} / \mathrm{m}$ to $45.4 \mathrm{~A} / \mathrm{m}$ when the AC current increased from $0.5 \mathrm{~mA}$ to $6 \mathrm{~mA}$, which also proves the decrease of circular magnetization in external DC field. Moreover, a sharp loop appeared when the driving current increased to $1 \mathrm{~mA}$ at $50 \mathrm{~Hz}$, indicating a much more sensitive circular permeability change with magnetic field. When the frequency was over $2 \mathrm{MHz}$, transverse Kerr 


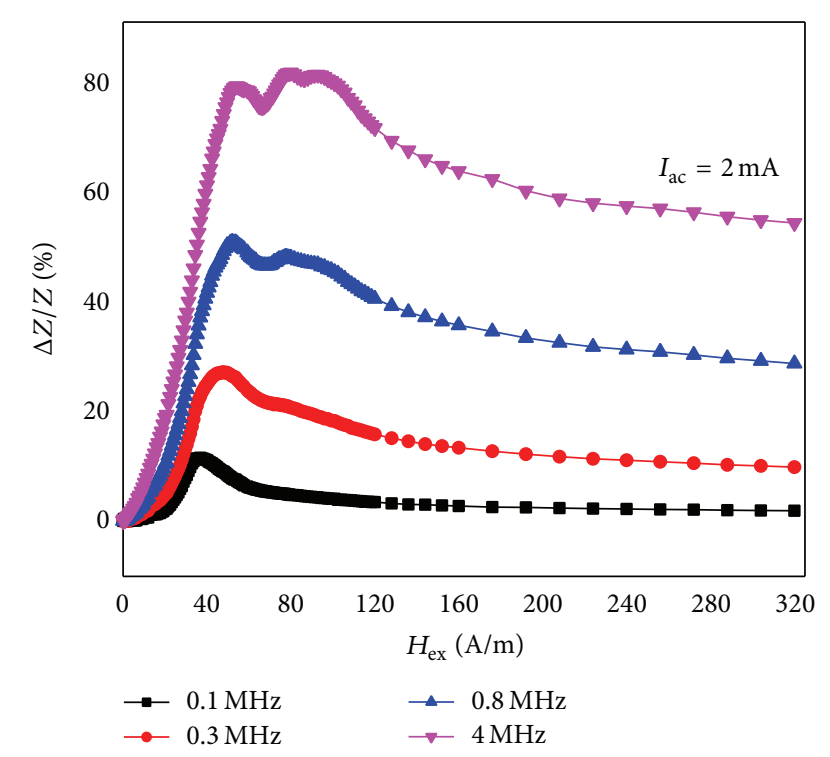

Figure 3: GMI profiles at different frequencies driven in $2 \mathrm{~mA}$.

intensity and coercivity had no clear change when the AC current increased from $0.5 \mathrm{~mA}$ to $20 \mathrm{~mA}$. In addition, there is no sensible difference observed between the hysteresis loops of different driving currents, as shown in Figure 4(b).

The AC driving current leads to a circumferential magnetic field $H_{\Phi}$ [8]:

$$
H_{\Phi}=\frac{I_{\mathrm{ac}} l}{\left(2 \pi r^{2}\right)},
$$

where $l$ and $r$ are the length and diameter of the measured microwire, respectively. The magnetic field amplitude $H_{\Phi}$ is associated with the rotational circular magnetization process [8]. In addition, the domain wall displacement mainly depends on DC magnetization. Furthermore, its displacement is the main magnetization process at relatively lower frequencies and the skin depth is large enough to penetrate the whole wire. The induced circular field by driving current then distributes in the total wire. The induced circumferential field gets stronger with the increase of driving current and with enhanced circular rotational magnetization. Circular magnetization under external magnetic field with circular permeability relatively decreases. As a consequence, GMI response decreases with the driving current at $0.1 \mathrm{MHz}$, as shown in Figure 5(a).

The skin effect gets stronger with the current frequency and displacement magnetization process and gradually gets strong in the magnetization process. The circumferential components of magnetic permeability depend on the induced circular magnetic field $H_{\Phi}$ and axial magnetic field. Larger $H_{\Phi}$ enhances a part of circular magnetization which is driven by this induced field and reduces the other part of circular magnetization which is driven by external magnetic field $H_{\text {ex }}$ [14]. The total impedance is originated from the axial external magnetic field and is also decided by circumferential magnetic permeability $\mu_{\Phi}$. However, components of the magnetic permeability tensor are dependent on axial external and induced circular magnetic field [8]. When the circular magnetic field reaches at around the circular coercive field value, $\mu_{\Phi}$ reaches the maximum values [8]. The existence of current $I_{p}$ which increases with frequency is correlated with circular coercive field, indicating that GMI ratio can be optimized by choosing an adequate value of driving current $I_{p}$ below $2 \mathrm{MHz}$.

Furthermore, the magnetization process also changes from the displacement of the domain walls to the rotation of magnetic moment $[1,15]$, with gradually increasing frequency. At a certain frequency, a balance between displacement magnetization and rotational process exists, during which a fluctuation of circular permeability appears. And from domain wall observation, there are different kinds of domains at lower external magnetic field firstly, but when the external field gets strong enough, domain structure transformation and annihilation will occur [16]. As a consequence, multipeak GMI feature expresses the driving current and frequency dependency.

In a range of a relatively high frequency over $1 \mathrm{MHz}$, GMI can roughly be described by the well-known standard skin effect [17]. The latter manifests that the applied current effectively flows in an outer part of the cross section of the microwire. The penetration depth, $\delta$, which measures this effect, is described by a commonly known expression derived under the assumption that the dimension of the wire in the magnetic field penetration direction is much larger than the penetration depth, expressed as $\delta / r$, where $r$ is the wire radius. Chizhik et al. developed a sample model to estimate the penetration depth as a function of experimentally obtainable ratio $R_{\mathrm{DC}} / R_{\mathrm{AC}}$ [14], where $R_{\mathrm{DC}}$ is the DC resistance of the wire and $R_{\mathrm{AC}}$ is the real component of impedance measured at a given frequency as a function of axially applied external magnetic field. The penetration depth is given as the following function [17]:

$$
\delta=r\left[1-\sqrt{1-\frac{R_{\mathrm{DC}}}{R_{\mathrm{AC}}}}\right] .
$$

In our data, $R_{\mathrm{AC}}$ is selected when the external field is zero. Equation (5) expresses the ratio $\delta / r$ of the penetration depth and the wire radius with various driving currents and frequencies. The ratio $\delta / r$ decreases with the increase of driving current $I_{\mathrm{ac}}$ and frequencies, which reflects that the skin effect is stronger as shown in Figure 6. Based on the equation of skin depth, skin effect is also enhanced with increasing frequency and circular magnetic permeability. Therefore, GMI effect is enhanced, as shown in Figure 5(b).

In this frequency range, the concentration of induced circumferential field gradually migrates from the total wire to the outer shell, which is beneficial for circular rotational magnetization, stronger circular permeability, and much more sensitive GMI effect, as observed in Figure 3. The basic mechanisms of magnetization reversal process which is dependent on the magnetic field would be different with frequency $[13,18]$. As a consequence, the domain wall motion and domain structure transformation in the functioning of sensor elements using magnetic microwires are both dependent on the driving current. Consequently, though the increase in 


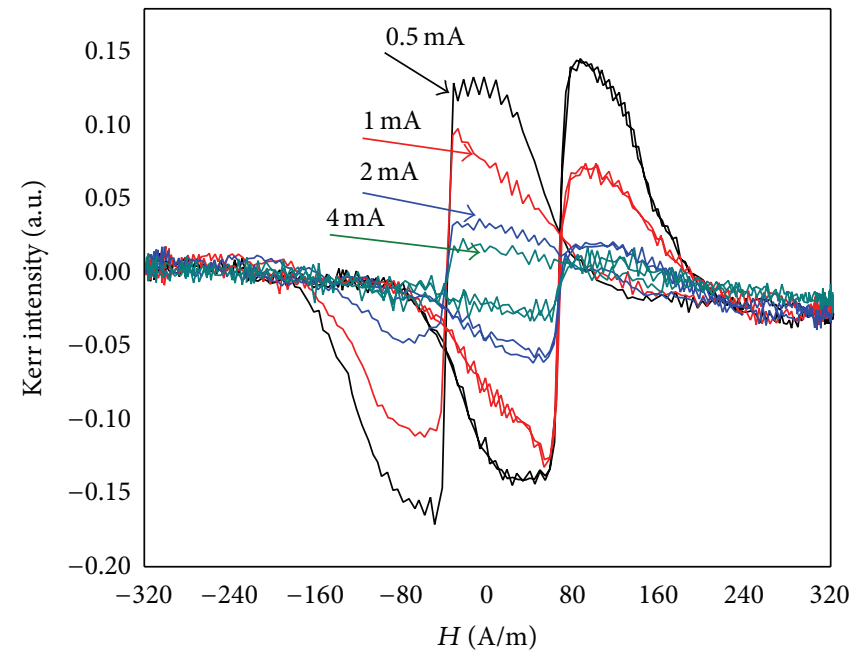

(a)



(b)

Figure 4: Transverse Kerr effect at (a) $50 \mathrm{~Hz}$ and (b) $10 \mathrm{MHz}$ of Co-based amorphous wires with different driving currents.

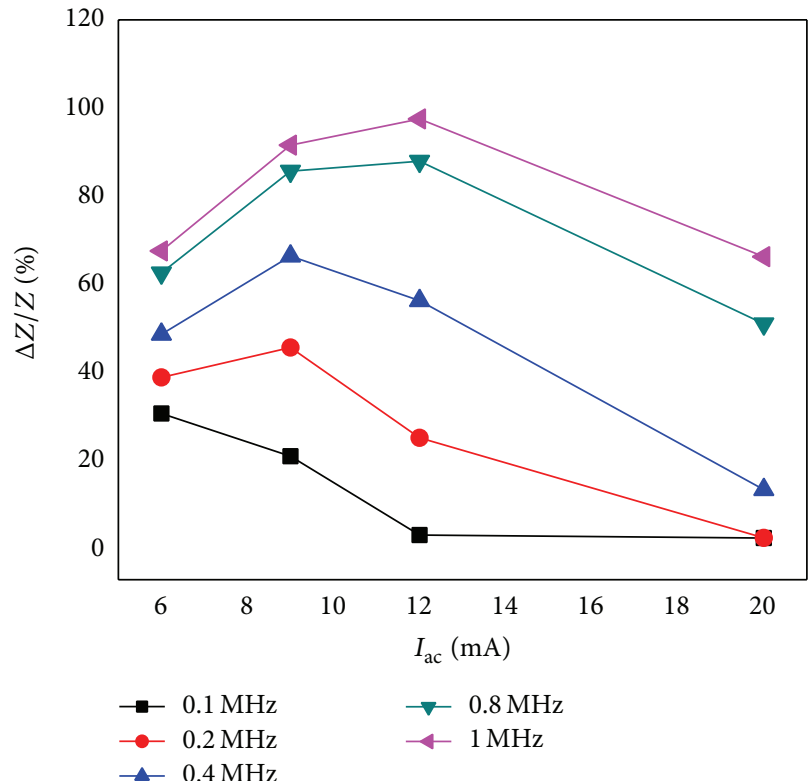

(a)

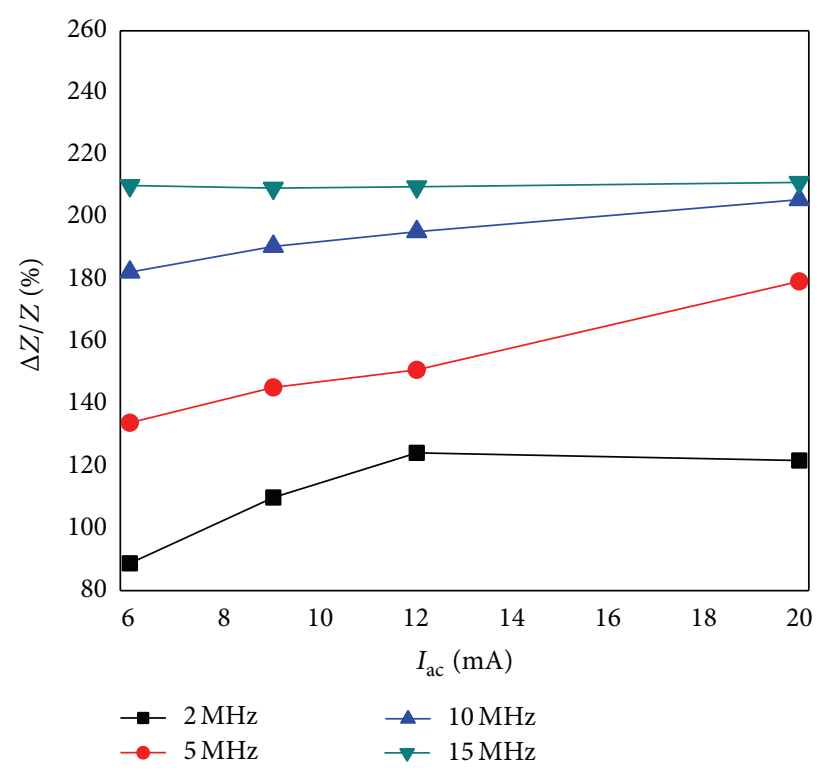

(b)

FIGURE 5: Driving current dependency on maximum impedance ratio $\Delta Z / Z$ (a) below $1 \mathrm{MHz}$ and (b) over $1 \mathrm{MHz}$.

driving current induces a stronger circumferential field, their distribution slightly has little effect compared with the strong skin effect when the frequency is up to several megahertz.

This evolution proves that the dependency of driving AC current on the GMI effect correlated with magnetization is a frequency function. When the frequency is lower than $2 \mathrm{MHz}$, circumferential magnetization in the DC field depends on the circular magnetic field induced by the driving current. However, the influence of AC current on magnetization weakens when the frequency is over $2 \mathrm{MHz}$ for strong skin effect, which is the key role in magnetization and GMI effect at higher frequencies.

\section{Conclusions}

The AC driving current influences the circular magnetization and the skin effect; thus, the difference in GMI response is observed. At lower frequencies $(<0.2 \mathrm{MHz})$, the increase in AC driving current reduced the rotational circular magnetization process under DC magnetic field, and the GMI response relatively decreased. Likewise, the balance between displacement magnetization and rotational process gives rises to multipeak GMI features with the increase of frequency. When it increases to megahertz, skin effect gets stronger and becomes the main factor in deciding the GMI response. 


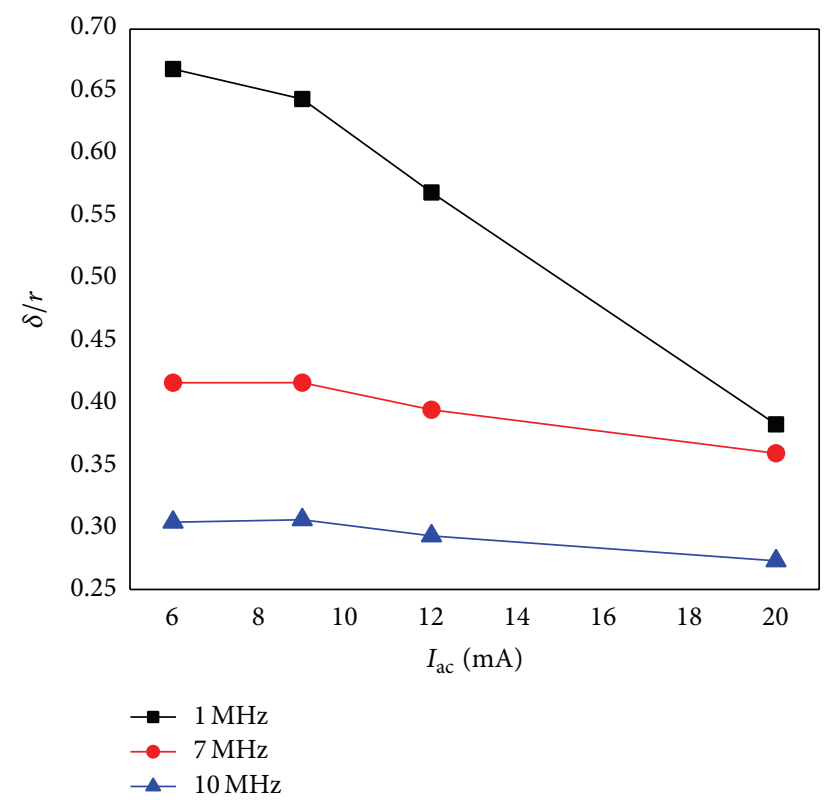

FIGURE 6: Skin effect at different driving currents.

\section{Conflict of Interests}

The authors declare that there is no conflict of interests regarding the publication of this paper.

\section{Acknowledgments}

The authors gratefully acknowledge Professor Julian Gonzalez and Dr. Alexander Chizhik for the Kerr effect measurements. And this project is supported by the Natural Science Foundation of Shanxi, China (Grant no. 2014021018-4), the State Key Lab of Advanced Metals and Materials (Grant no. 2013-Z06), Natural Science Funds, Ningxia University, China (Grant no. ZR1411), and Research Starting Funds for Imported Talents, Ningxia University, China (Grant no. BQD2014019).

\section{References}

[1] R. S. Beach and A. E. Berkowitz, "Giant magnetic field dependent impedance of amorphous FeCoSiB wire," Applied Physics Letters, vol. 64, no. 26, pp. 3652-3654, 1994.

[2] H. Chiriac, D.-D. Herea, and S. Corodeanu, "Microwire array for giant magneto-impedance detection of magnetic particles for biosensor prototype," Journal of Magnetism and Magnetic Materials, vol. 311, no. 1, pp. 425-428, 2007.

[3] A. Kumar, S. Mohapatra, V. Fal-Miyar et al., "Magnetoimpedance biosensor for $\mathrm{Fe}_{3} \mathrm{O}_{4}$ nanoparticle intracellular uptake evaluation," Applied Physics Letters, vol. 91, no. 14, Article ID 143902, 2007.

[4] G. Kurlyandskaya and V. Levit, "Magnetic Dynabeads detection by sensitive element based on giant magnetoimpedance," Biosensors and Bioelectronics, vol. 20, no. 8, pp. 1611-1616, 2005.

[5] Z. G. Liu, L. H. Chai, Y. Y. Chen, F. T. Kong, H. A. Davies, and I. A. Figueroa, "Microstructure evolution in rapidly solidified $Y$ added TiAl ribbons," Intermetallics, vol. 19, no. 2, pp. 160-164, 2011.

[6] D. Zhu, D. Dong, C. Ni et al., "Effect of wheel speed on the microstructure and nanohardness of rapidly solidified Ti-48Al2Cr alloy," Materials Characterization, vol. 99, pp. 243-247, 2015.

[7] G. V. Kurlyandskaya, "Giant magnetoimpedance for biosensing: advantages and shortcomings," Journal of Magnetism and Magnetic Materials, vol. 321, no. 7, pp. 659-662, 2009.

[8] P. Aragoneses, A. P. Zhukov, J. Gonzalez, J. M. Blanco, and L. Dominguez, "Effect of AC driving current on magnetoimpedance effect," Sensors and Actuators A: Physical, vol. 81, no. 1, pp. 86-90, 2000.

[9] A. P. Chen, M. R. Britel, V. Zhukova et al., "Influence of AC magnetic field amplitude on the surface magnetoimpedance tensor in amorphous wire with helical magnetic anisotropy," IEEE Transactions on Magnetics, vol. 40, no. 5, pp. 3368-3377, 2004.

[10] L.-Y. Feng, F.-S. Qin, and W.-J. Yong, "Influence of AC current on the profile of GMI effect in Fe-based nanocrystalline wire," Acta Physica Sinica, vol. 60, no. 3, Article ID 037306, 2011.

[11] H. Chiriac, M. Tibu, A.-E. Moga, and D. D. Herea, "Magnetic GMI sensor for detection of biomolecules," Journal of Magnetism and Magnetic Materials, vol. 293, no. 1, pp. 671-676, 2005.

[12] J.-F. Sun, J.-S. Liu, D.-W. Xing, and X. Xue, "Experimental study on the effect of alternating-current amplitude on GMI output stability of Co-based amorphous wires," Physica Status Solidi A, vol. 208, no. 4, pp. 910-914, 2011.

[13] A. Zhukov, A. Talaat, M. Ipatov, J. M. Blanco, and V. Zhukova, "Tailoring of magnetic properties and GMI effect of Co-rich amorphous microwires by heat treatment," Journal of Alloys and Compounds, vol. 615, pp. 610-615, 2014.

[14] A. Chizhik, A. Zhukov, J. Gonzalez, and J. M. Blanco, "Effect of high-frequency driving current on magnetization reversal in Co-rich amorphous microwires," Applied Physics Letters, vol. 85, no. 12, pp. 2292-2294, 2004.

[15] S.-L. Zhang, D.-W. Xing, and J.-F. Sun, "Influence of the distance between two voltage contacts on giant magneto-impedance of Co-based amorphous wires," Chinese Physics B, vol. 19, no. 7, Article ID 077502, 2010.

[16] A. Chizhik, A. Stupakiewicz, A. Zhukov, A. Maziewski, and J. Gonzalez, "Experimental demonstration of basic mechanisms of magnetization reversal in magnetic microwires," Physica B: Condensed Matter, vol. 435, pp. 125-128, 2014.

[17] H. K. Lachowicz, K. L. Garcia, M. Kuźmiński, A. Zhukov, and M. Vázquez, "Skin-effect and circumferential permeability in micro-wires utilized in GMI-sensors," Sensors and Actuators A: Physical, vol. 119, no. 2, pp. 384-389, 2005.

[18] S. H. Whang and Z. X. Li, "Tetragonal distortion and its relaxation in rapidly quenched $\mathrm{L}_{0} \mathrm{TiAl}$ compounds," Materials Science and Engineering, vol. 98, pp. 269-272, 1988. 


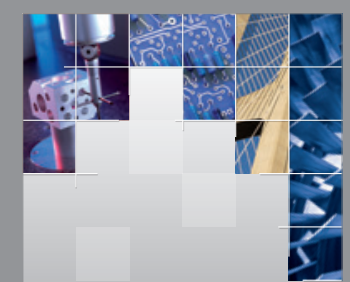

\section{Enfincering}
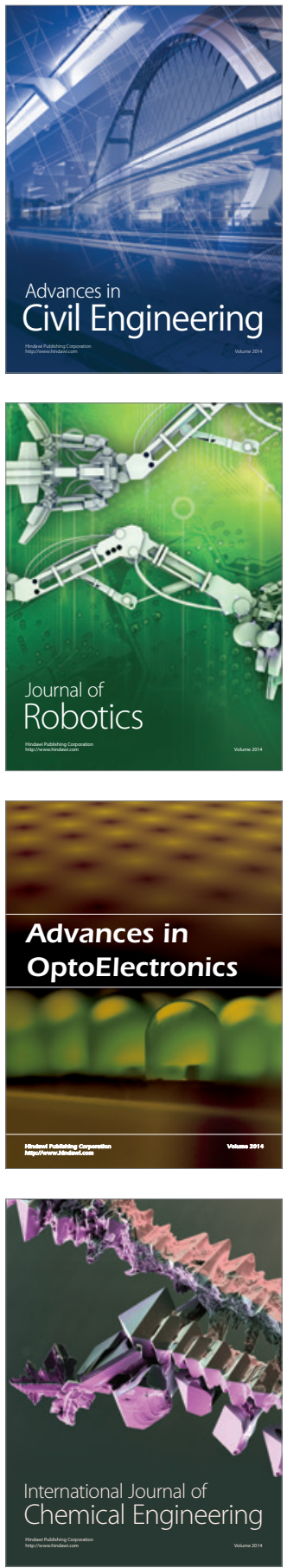

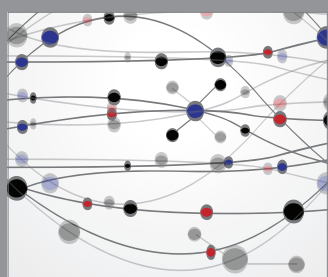

The Scientific World Journal

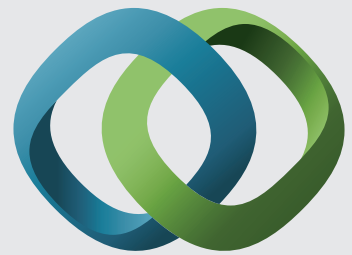

\section{Hindawi}

Submit your manuscripts at

http://www.hindawi.com
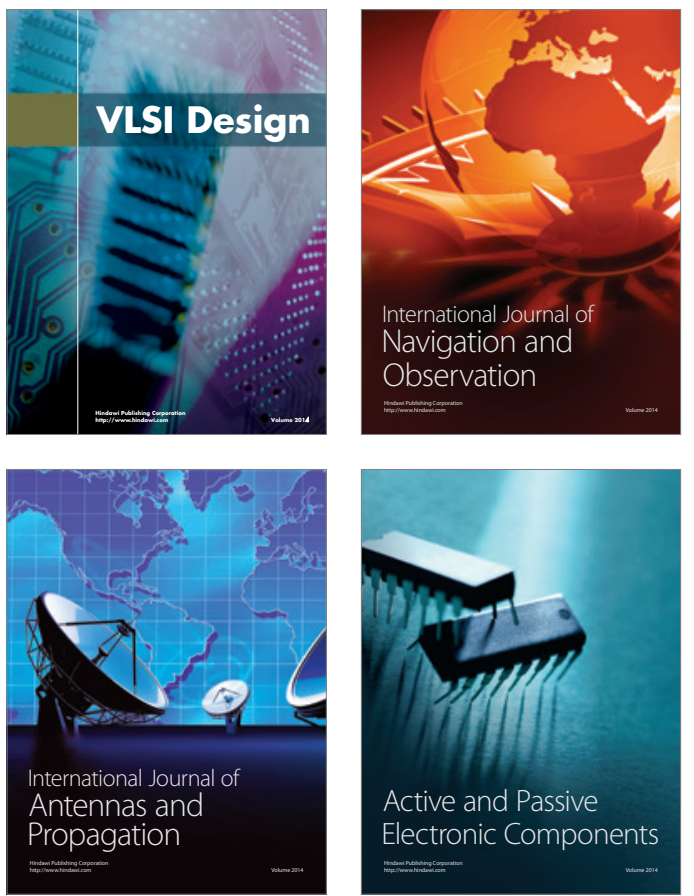
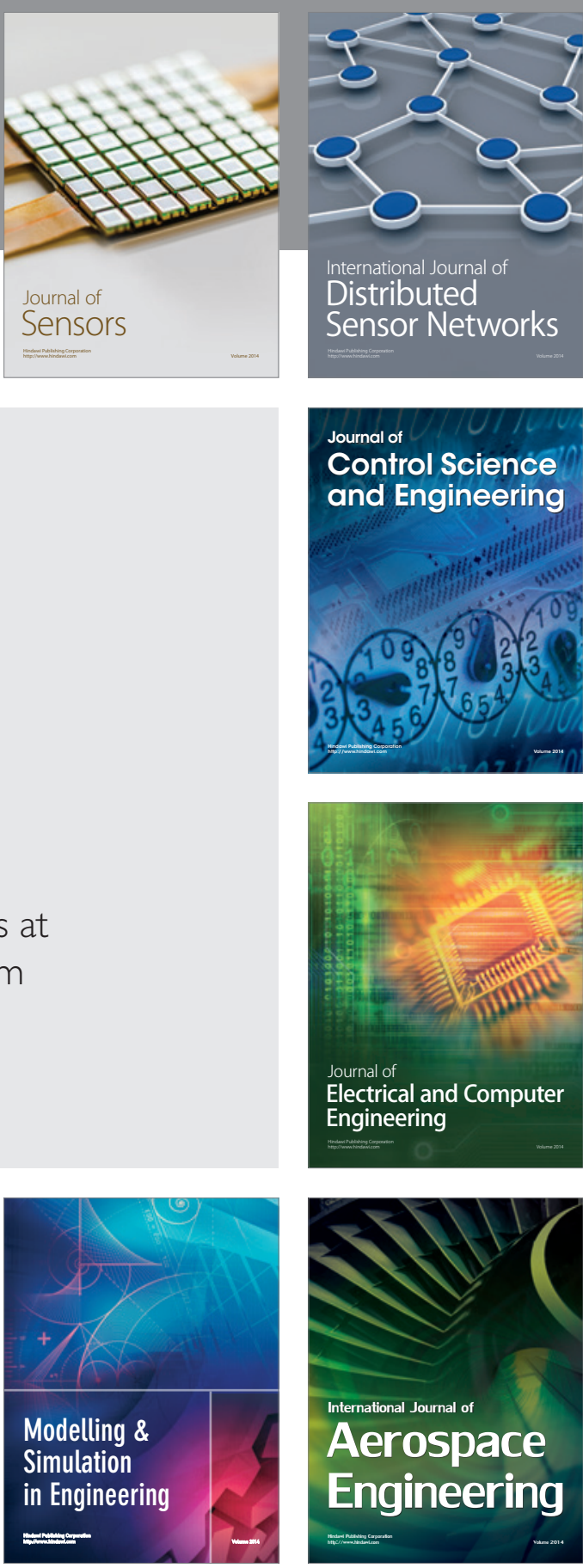

International Journal of

Distributed

Sensor Networks

Journal of

Control Science

and Engineering
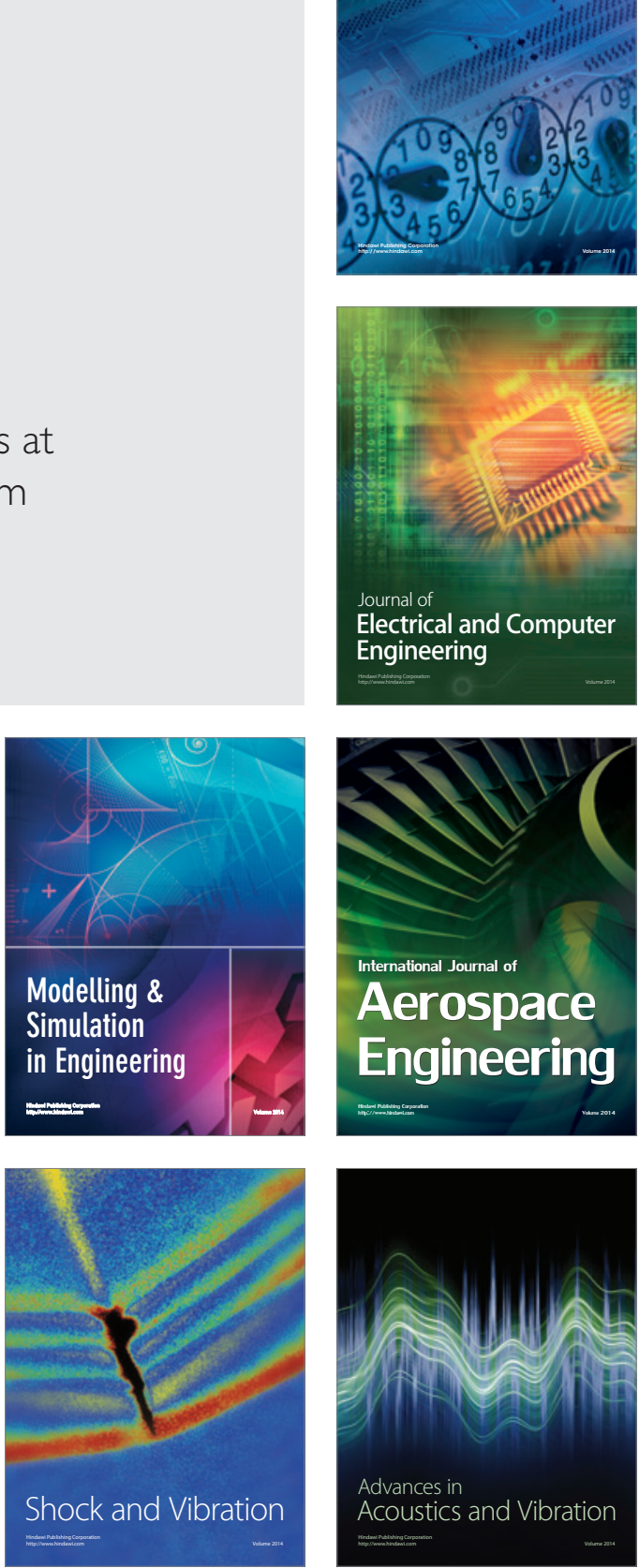